\title{
ON A THEOREM OF HAAR
}

\section{J. J. PRICE}

Let $\left\{\phi_{n}\right\}$ be an orthonormal set in $L^{2}[0,1], \phi_{n}$ having finite total variation $v_{n}$. Haar proved [1] that $\lim v_{n}=\infty$ and that consequently $\left\{\phi_{n}\right\}$ has an intrinsic ordering according to nondecreasing total variation. He showed, in fact, that in this ordering $v_{n}>c \sqrt{n}$ where $c$ is an absolute constant. A different proof was later given by Rudin [2].

We present a short proof that $\lim v_{n}=\infty$ under a much weaker hypothesis than orthonormality.

THEOREM. Let $\left\{\phi_{n}\right\}_{1}^{\infty}$ be a set of functions in $L^{2}[0,1]$ such that for each $n,\left\|\phi_{n}\right\|=1$ and $\phi_{n}$ has finite total variation $v_{n}$. If

$$
\limsup _{n} \limsup _{k}\left(\phi_{n}, \phi_{k}\right)<1,
$$

then

$$
\lim v_{n}=\infty .
$$

Proof. Assume (2) is false. Then there is a subsequence $\left\{\phi_{n_{i}}\right\}$ and a constant $M$ such that $v_{n_{i}}<M$ for each $i$. Since

$$
\sup _{x} \phi_{n_{i}}(x)-\inf _{x} \phi_{n_{i}}(x) \leqq v_{n_{i}}<M,
$$

it follows that $\left|\phi_{n_{i}}(x)\right| \leqq M+1$ for all $x$. For, if $\sup _{x} \phi_{n_{i}}(x)>M+1$, then

$$
\inf _{x} \phi_{n_{\mathrm{i}}}(x)>\sup _{x} \phi_{n_{\mathrm{i}}}(x)-M>1
$$

which is incompatible with the hypothesis $\left\|\phi_{n_{i}}\right\|=1$. Similarly, $\inf _{x} \phi_{n_{i}}(x)>-(M+1)$.

Thus the functions $\phi_{n_{i}}$ are uniformly bounded and their total variations are bounded. By Helly's Theorem, there is a convergent subsequence $\left\{\phi_{n_{i_{k}}}\right\}$.

$$
\begin{aligned}
\left(\phi_{n_{i j}}, \phi_{n_{i_{k}}}\right) & =\frac{1}{2}\left(\left\|\phi_{n_{i_{j}}}\right\|{ }^{2}+\left\|\phi_{n_{i_{k}}}\right\|^{2}-\left.\left\|\phi_{n_{i_{j}}}-\phi_{n_{i_{k}}}\right\|\right|^{2}\right) \\
& =1-\frac{1}{2}\left\|\phi_{n_{i_{j}}}-\phi_{n_{i_{k}}}\right\|^{2} .
\end{aligned}
$$

By bounded convergence, $\left\|\phi_{n_{i j}}-\phi_{n_{i k}}\right\|^{2} \rightarrow 0$ as $j, k \rightarrow \infty$. Therefore, given $\epsilon$,

$$
\left(\phi_{n_{i j}}, \phi_{n_{i_{k}}}\right)>1-\epsilon
$$

Received by the editors October 4, 1966. 
for all sufficiently large values of $j$ and $k>j$. Hence,

$$
\lim \sup \left(\phi_{n_{i j}}, \phi_{k}\right)>1-\epsilon
$$

for infinitely many indices $n_{i_{j}}$. Since $\epsilon$ was arbitrary,

$$
\limsup _{n} \limsup _{k}\left(\phi_{n}, \phi_{k}\right)=1 \text {. }
$$

But this contradicts hypothesis (1) and the theorem is proved.

Haar's result that $v_{n}>c \sqrt{n}$ for an orthonormal set can also be extended.

Theorem. If

$$
\sum_{1 \leq i<j<\infty}^{\infty}\left|\left(\phi_{i}, \phi_{j}\right)\right|<\infty
$$

then $\left\{\phi_{n}\right\}$ can be ordered according to nondecreasing total variations so that $v_{n}>a \sqrt{n}$ where $a$ is a constant depending only on the sum (3).

For the proof, one need only modify Haar's original argument by using in place of the Bessel inequality the inequality

$$
c_{1}^{2}+c_{2}^{2}+\cdots+c_{n}^{2} \leqq b\|f\|^{2}, \quad c_{i}=\left(f, \phi_{i}\right)
$$

which holds under hypothesis (3).

Condition (1) was suggested by the referee to replace our somewhat stronger hypothesis, $\lim \sup _{n, k}\left(\phi_{n}, \phi_{k}\right)=1$.

\section{REFERENCES}

1. A. Haar, Über einige Eigenschaften der orthogonalen Funktionensysteme, Math. Z. 31 (1930), 128-137.

2. Walter Rudin, $L^{2}$-approximation by partial sums of orthogonal developments, Duke Math. J. 19 (1952), 1-4.

Institute for Advanced Study 